(n)

\title{
Gestational diabetic transcriptomic profiling of microdissected human trophoblast
}

\author{
Muhammad Furqan Bari1,*, Sherry Ngo ${ }^{2, *}$, Claire C Bastie ${ }^{3}$, Allan M Sheppard ${ }^{2}$ \\ and Manu Vatish 4 \\ 'Department of Pathology, Dow International Medical College, Karachi, Pakistan \\ ${ }^{2}$ Liggins Institute, University of Auckland, Auckland, New Zealand \\ 3Division of Biomedical Sciences, Warwick Medical School, Coventry, UK \\ ${ }^{4}$ Nuffield Department of Obstetrics and Gynaecology, University of Oxford, Oxford, UK \\ (*M F Bari and S Ngo contributed equally to this work)
}

Correspondence

should be addressed

to A M Sheppard or

M Vatish

Emails

a.sheppard@auckland.ac.nz or manu.vatish@obs-gyn.ox.ac.uk

\begin{abstract}
Gestational diabetes mellitus (GDM), the most common metabolic complication of pregnancy, is influenced by the placenta, and its prevalence directly increases with obesity. Therefore, to define the aetiology of GDM requires that the confounding influence of obesity and the heterogeneous nature of the placenta impairing accurate quantitative studies be accounted for. Using laser capture microdissection (LCM), we optimized RNA extraction from human placental trophoblast, the metabolic cellular interface between mother and foetus. This allowed specific transcriptomic profiling of trophoblast isolated from GDM, and obese and normal human placentae. Genome-wide gene expression analysis was performed on the RNA extracted from the trophoblast of GDM and obese and normal placentae. Forty-five differentially expressed genes (DEGs) specifically discriminated GDM from matched obese subjects. Two genes previously linked with GDM, pregnancy specific beta-1 glycoprotein 6 (PSG6) and placental system A sodium-dependent transporter system (SLC38A1), were significantly increased in GDM. A number of these DEGs (8 ubiquitin-conjugating enzymes (UBE) splice variants (UBE2D3 variants $1,3,4,5,6,7$, and 9) and UBE2V1 variant 4)) were involved in RNA processing and splicing, and a significant number of the DEGs, including the UBE variants, were associated with increased maternal fasting plasma glucose. It is concluded that DEGs discriminating GDM from obese subjects were pinpointed. Our data indicate a biological link between genes involved in RNA processing and splicing, ubiquitination, and fasting plasma glucose in GDM taking into account obesity as the confounder.
\end{abstract}

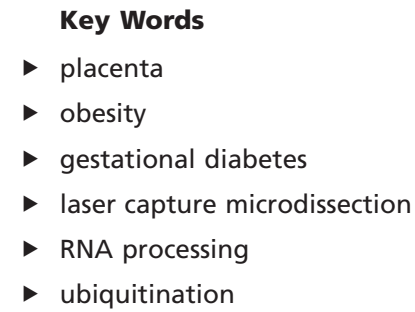

\section{Introduction}

Gestational diabetes mellitus (GDM), defined as glucose intolerance resulting in hyperglycaemia of variable severity during pregnancy, is the commonest metabolic complication of pregnancy (Virjee et al. 2001), with increasing prevalence directly linked to increasing obesity in women of reproductive age (Dabelea et al. 2005). Women with GDM and obesity (and their offspring) have a higher prevalence of metabolic syndrome than those 
without a history of GDM or obesity (Catalano et al. 2012; Vohr \& Boney 2008) as well increased GDM recurrence in subsequent pregnancies (Bottalico 2007). Approximately $50 \%$ of women with GDM are expected to develop type 2 diabetes within 5-10 years of delivery (Bellamy et al. 2009). Other risks of GDM include an increased likelihood of pregnancy complications, such as pre-eclampsia and offspring metabolic syndrome (Boney et al. 2005; Bryson et al. 2003).

GDM is linked with aberrant placental function and a number of placenta-derived molecules implicated in the manifestation of insulin resistance associated with GDM (Desoye \& Hauguel-de Mouzon 2007). The key functioning unit of the placenta is the chorionic villus, in which a multi-nucleated trophoblast (comprising of syncytiotrophoblast and cytotrophoblast) lies in direct contact with maternal blood and remains the primary site of metabolic exchange at the maternal-foetus interface (Huppertz 2008). In addition to the trophoblast, however, there is an underlying stromal tissue and foetal capillaries as well as placental macrophages (known as Hofbauer cells) (Huppertz 2008). The heterogeneous nature of this tissue has rendered quantitative studies of the trophoblast difficult. While flow cytometry and cell culture of cellular fractions can be used to examine other cell types within the placenta, the trophoblast (part of which is a true syncytium) is not amenable to these techniques without contamination. This issue can be avoided by using LCM, a technique that allows isolation of distinct cell types (in our case, trophoblast) from frozen section or formalin-fixed samples of tissue. Studies have been hampered both by the difficulties of separating the effects of obesity from those of GDM and by extracting RNA of sufficient quality to perform meaningful downstream analysis. Thus our data allows the effects of obesity to be subtracted from GDM at the level of the trophoblast with high quality RNA subjected to microarray.

Our findings indicate that GDM placental trophoblast is fundamentally, biologically distinct from the states of normality and obesity at gene expression levels. Taking into account obesity as the confounder, genes that showed increased expression in GDM were largely involved in RNA processing and splicing. We also found increased expression of multiple splice variants of the ubiquitin-conjugating enzymes UBE2D3, and UBE2V1 variant 4, in GDM. The expression of these genes was significantly associated with maternal fasting plasma glucose, suggesting an association of these genes with glucose intolerance, a hallmark of GDM.

\section{Materials and methods}

\section{Samples}

All study participants were pregnant women scheduled for elective Caesarean section at 39-40 weeks of gestation. The indication for Caesarean section was either breech presentation or repeat Caesarean section. Oral glucose (75g) tolerance tests (OGTTs) were carried out at 26-28 weeks of gestation in all participants to diagnose or exclude GDM. All GDM patients were well controlled on insulin and only those with male offspring were recruited. The Coventry Local Research Ethics committee approved the study, and all patients gave written informed consent (Research Ethics Committees 07/H1210/118). Women with multiple pregnancies, as well as patients with cardiovascular disease, pre-eclampsia, or other relevant diseases were excluded. Placental biopsies were taken in the operating theatre and immediately snap-frozen in liquid nitrogen and transferred to a $-80^{\circ} \mathrm{C}$ freezer until needed. Placental biopsies (by a random sampling method (Burton et al. 2014)) from four lean (normal; Norm), four obese (Ob), and four gestational diabetic (GDM) subjects were used.

\section{Laser microdissection (LCM): efficacy of staining and duration of LCM procedure on RNA Integrity Number (RIN)}

The sections of $8 \mu \mathrm{m}$ thickness were cut using a cryostat (Leica Microsystems, Milton Keynes, UK) at $-25^{\circ} \mathrm{C}$ and adhered onto MembraneSlides (Molecular Machines \& Industries AG, Glattbrugg, Switzerland) pre-cooled inside the cryostat and maintained dehydrated in $-80^{\circ} \mathrm{C}$ freezer until needed. All slides, LCM caps, and solutions were obtained from Molecular Machines \& Industries. The slides were dehydrated in graded alcohols and xylene and placed in a desiccator until laser capture. The sections were stained with (a) Acridine orange (Life Technologies), (b) Alcoholic cresyl violet (Life Technologies), (c) Alcoholic cresyl violet and alcoholic eosin Y (Sigma-Aldrich), (d) Arcturus Paradise stain (Life Technologies) in combination with RNAse inhibitor, (e) Arcturus Paradise stain, (f) haematoxylin (Leica Microsystems, Milton Keynes, UK) and alcoholic eosin Y in combination with RNAse inhibitor, and (g) haematoxylin and alcoholic eosin Y together with alcoholic eosin-Y. RIN values were assessed using Nano/Pico Chips on an Agilent 2100 Bioanalyzer (Agilent Technologies Inc.) (Clement-Ziza et al. 2008). Having identified the optimal stain, LCM was performed in 0,30 and 50 min to assess the effect of duration of LCM on RIN values.

Published by Bioscientifica Ltd. 


\section{RNA extraction and gene expression profiling}

The RNA was extracted using RNeasy Micro Kit (Qiagen Inc.) for the analysing RIN on the Bioanalyzer, while for gene expression profiling, microdissected samples were exposed to Prelude Direct Lysis Module (NuGEN, CA, USA) and hybridized to Affymetrix HuGene ST 1.0 arrays using manufacturer's protocols (Affymetrix Inc.). One chip was used for each clinical sample. Raw intensity data files (.CEL) were read using the publicly available oligo package in $\mathrm{R}$ (Carvalho et al. 2007). Background subtraction, quantile normalization and summarization via median-polish, and finally $\log 2$ transformed were performed on the raw data using the R package RMA (Robust Multichip Average preprocessing methodology) (Irizarry et al. 2003). Analysis of differential gene expression using a linear models approach was performed using the R package Limma (Smyth 2004). Adjusted $P$-values $<0.05$ using FDR correction were considered statistically significant. Results were filtered using ranked two-fold change in gene expression from pairwise comparisons between the three subject groups (Norm, Ob and GDM). Heatmap illustration of sample hierarchical clustering was generated using the $\mathrm{R}$ package gplots (http://www.cran.r-project.org/).

\section{Validation of gene expression microarray data using qRT-PCR}

The four genes that were upregulated by two-fold in GDM group compared with $\mathrm{Ob}$ and Norm groups were selected for validating the gene expression microarray data in the same four samples for each group as used for gene expression microarrays. All experiments were carried out in triplicate. The genes and primers sequences used in this study are UBE2V1 (Forward: CAAGAGCGACGCAAG ATG; Reverse: TCTGGGTATTTAGGTCCACATT); UBE2D3 transcript variant 6 (Forward: GCAGAAAGGATACTGT GTG; Reverse: CAGGATTGTCTCGTCTCA); UBE2D3 transcript variant 5 (Forward: CATTTTGGAGGCGGA ACC; Reverse: AACTTCGTGGCTGGCTAA), and PSG6 Forward: CCTTACATCACCATCAACAA; Reverse: TCCGA CTCTTAGGTTCAC). All the primers were designed as described previously (Bari et al. 2013). RNA from laser microdissected samples was extracted using the Arcturus PicoPure RNA Isolation Kit (Life Technologies) with on-column DNase digestion (Qiagen) and converted to cDNA using High-Capacity cDNA Reverse Transcription Kit (Life Technologies) as recommended by the manufacturer. Genomic DNA was digested using recombinant shrimp DNase (Affymetrix). The qRT-PCR was performed using SYBR green-based chemistry on an Applied Biosystems 7500 Fast PCR system using Fast SYBR green master mix (Life Technologies), following the manufacturer's recommendations. The qRT-PCR data were analysed using DataAssist software ver 3.01 (Life Technologies), $18 \mathrm{~S}$ was taken as endogenous control, and $P$-values were adjusted using Benjamini-Hochberg false discovery rate.

\section{Statistical analysis}

All statistical analysis was performed in R (http://www. bioconductor.org/). Assessment of statistically significant difference in clinical measures between subject groups was performed using Tukey method of multiple comparisons test. Linear regression analysis was performed to determine association of gene expression with maternal fasting plasma glucose using deltaGT. 'deltaGT' was calculated as [Glucose $]_{2 \mathrm{~h}}-[\text { Glucose }]_{\text {Baseline }}$ measured using oral glucose tolerance test. KEGG pathway data base is available at http://www.genome.jp/kegg/pathway.html

\section{Results}

\section{Maternal and foetal clinical characteristics}

The subjects used in this study averaged 39 weeks of gestation at delivery (Table 1 ). The BMI for Norm group $\left(24.1 \pm 1.0 \mathrm{~kg} / \mathrm{m}^{2}\right)$ was significantly lower than those in GDM $\quad(32.3 \pm 0.8 \mathrm{~kg} ; \quad P=0.0001) \quad$ or $\quad \mathrm{Ob} \quad(30.8 \pm 0.3$; $P=0.0003)$ groups. Baseline glucose levels were similar between all subjects (GDM: $5.3 \pm 2 \mathrm{mM}$; Norm: $5 \pm 0.2 \mathrm{mM}$; Ob: $5.3 \pm 0.1 \mathrm{mM}$ ). As expected, fasting plasma glucose following an oral glucose tolerance test (OGTT) $(8.6 \pm 0.3 \mathrm{mM})$ was significantly increased in GDM subjects compared with Norm $\left(5.5 \mathrm{mM} ; P=3.2 \times 10^{-6}\right)$ or Ob subjects $\left(5.6 \pm 0.1 \mathrm{mM} ; P=3.2 \times 10^{-6}\right)$. Maternal insulin resistance has been recently reported to correlate with foetal insulin resistance (Wang et al. 2013). In our study, maternal insulin level was mildly elevated in GDM $(25 \pm 12.3 \mu \mathrm{IU} / \mathrm{mL})$ and $\mathrm{Ob}(22 \pm 3.5 \mu \mathrm{IU} / \mathrm{mL})$ groups compared with Norm $(14 \pm 8.1 \mu \mathrm{IU} / \mathrm{mL})$ group, albeit not significant. Maternal glucose was mildly elevated in the GDM $(4.8 \pm 0.3 \mathrm{mM})$ compared with $\mathrm{Ob}(4.3 \pm 0.2 \mathrm{mM})$ or Norm $(4.4 \pm 0.1 \mathrm{mM})$ group. Foetal insulin level was lower in GDM $(6.1 \pm 0.7 \mu \mathrm{IU} / \mathrm{mL})$ group compared with Norm $(12.4 \pm 1.2 \mu \mathrm{IU} / \mathrm{mL} ; P=0.11)$ or Ob $(15.8 \pm 4.4 \mu \mathrm{IU} /$ $\mathrm{mL} ; P=0.07$ ) group. Foetal glucose was mildly elevated in GDM $(4.6 \pm 0.3 \mathrm{mM})$ group compared with Norm

Published by Bioscientifica Ltd. 
ชิ

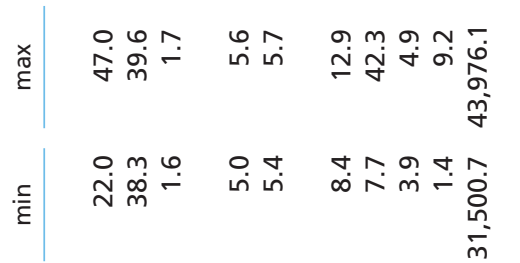

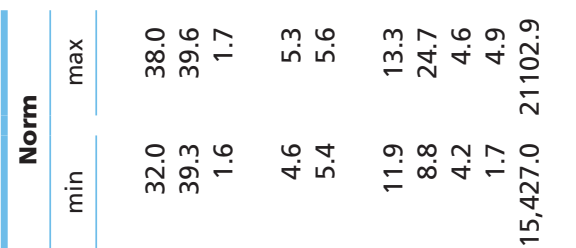

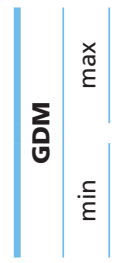

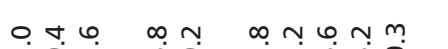

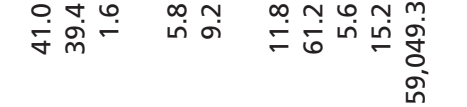

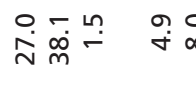

+

는 눈

$\stackrel{\sim}{\stackrel{N}{N}} \underset{m}{m}$

ن்

$-m m$.

뭉

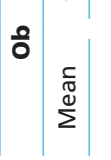

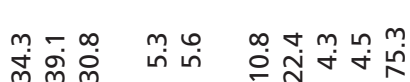

mim

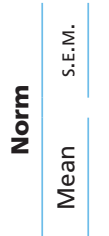

$\stackrel{m}{-} \div \stackrel{0}{\circ} \stackrel{-}{-}$

ํํㅇํำ

min

岗官方

ํํ เกำ

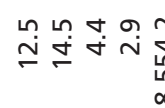

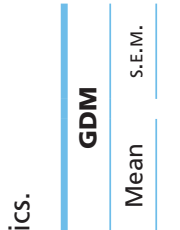

$0 m \infty \sim$

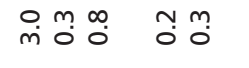

$\infty 0 m m$

$m$
$n \rightarrow \infty$
$\infty$

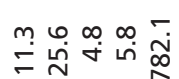

m.

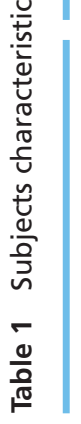

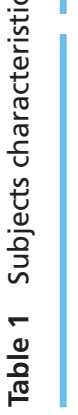

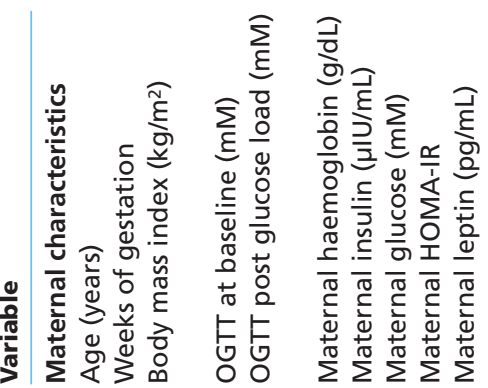

http://joe.endocrinology-journals.org

C 2016 Society for Endocrinology Printed in Great Britain

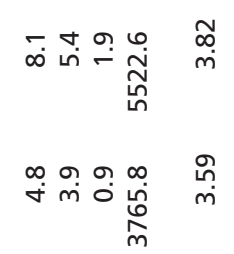

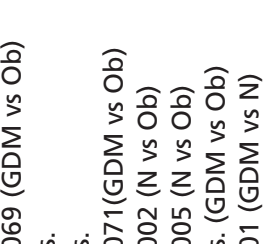

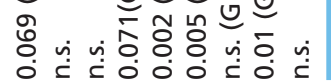

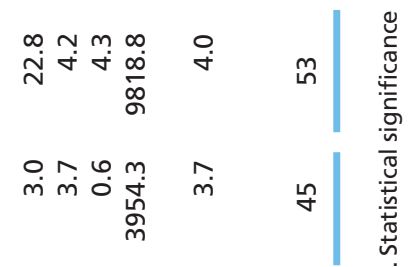

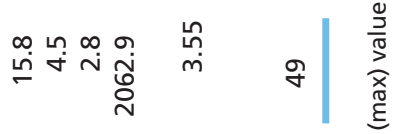

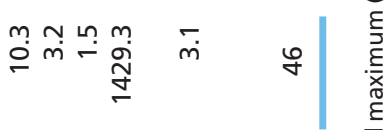

†ं大்

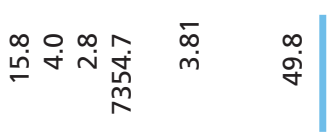

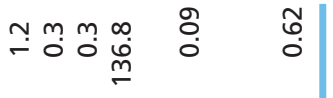

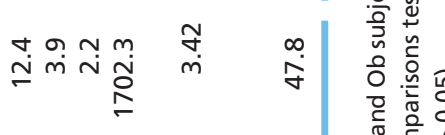

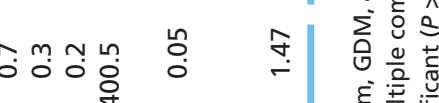

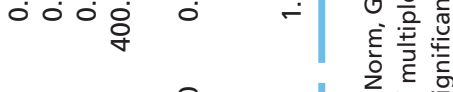

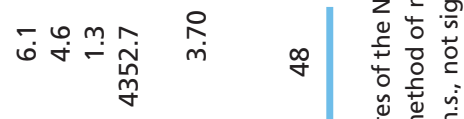

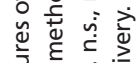

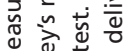

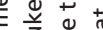

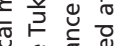

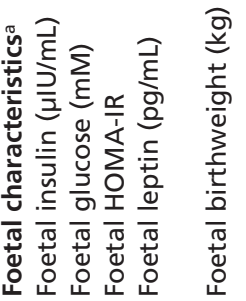

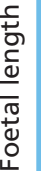


$(3.9 \pm 0.3 \mathrm{mM})$ or $\mathrm{Ob}(4.0 \pm 0.1 \mathrm{mM})$ group, but was not statistically significant. We found maternal leptin was significantly elevated in GDM $(44,782.1 \pm 5407.3 \mathrm{pg} / \mathrm{mL}$; $P=0.001)$ and $\mathrm{Ob}(38,175.3 \pm 2647.7 \mathrm{pg} / \mathrm{mL} ; \quad P=0.01)$ compared groups with Norm $(18,554.2 \pm 1289.3 \mathrm{pg} / \mathrm{mL})$ group. Similarly, foetal leptin was significantly elevated in GDM $(4352.7 \pm 400.5 \mathrm{pg} / \mathrm{mL})$ and $\mathrm{Ob}$ $(7354.7 \pm 1371.0 \mathrm{pg} / \mathrm{mL})$ groups compared with Norm $(1702.3 \pm 136.8 \mathrm{pg} / \mathrm{mL})$ group.

\section{Acridine orange and alcoholic cresyl violet stains are most effective in preserving human placental trophoblast RNA}

LCM was performed as described above (Fig. 1). The tissues processed for RNA with all steps of hydration and dehydration without any stain yielded a RNA Integrity Number (RIN) of 8.6 (Supplementary Figure 1, see section on supplementary data given at the end of this article). Staining with Acridine orange gave the highest RIN value of 8.5 (a drop of only 0.1 ; Supplementary Figure 1). However as the filter for this fluorescent dye is not available in all instruments, we used other non-fluorescent dyes as well. Among the non-fluorescent dyes, the alcoholic cresyl violet was the most effective dye in preserving RIN and yielded a RIN of 8.0 (drop of 0.6; Supplementary Figure 1). As cytoplasmic staining was also needed in dissecting the trophoblast, it was combined with alcoholic eosin Y, which dropped the RIN to 7.8 (drop of 0.8; Supplementary Figure 1). The Arcturus Paradise stain with the addition of RNase inhibitor at a concentration of 1 unit/ $\mu \mathrm{L}$ of stain yielded a RIN of 6.7 (drop of 1.9; Supplementary Figure 1), whereas the use of Arcturus Paradise stain alone dropped the RIN to 6.0 (drop of 2.6; Supplementary Figure 1). In contrast, the use of routine haematoxylin in combination with alcoholic eosin Y together with RNAse inhibitor yielded a RIN of 5.4 (drop of 3.2; Supplementary Figure 1) and finally haematoxylin with alcoholic eosin Y without the RNAse inhibitor dropped the RIN to 3.0 (drop of 5.6; Supplementary Figure 1). Having identified alcoholic cresyl violet and alcoholic eosin- $Y$ as the optimal nonfluorescent stain, we next assessed the effect of duration of LCM on RIN values.

\section{LCM within 30 min maintains RIN}

Time points at 0,30 , and 50 min were selected and resulting RIN values $7.5,6.9$, and 6.2 respectively (Supplementary Figure 2) indicated that the degradation is proportional to the duration of dissection; with 30 min being optimal to allow laser-capture to be performed while maintaining RNA integrity. Tissue extraction for microarray experiments was thus performed under $30 \mathrm{~min}$ in tissues stained with alcoholic cresyl violet and alcoholic eosin-Y. The tissues destined for microarray were placed directly in the
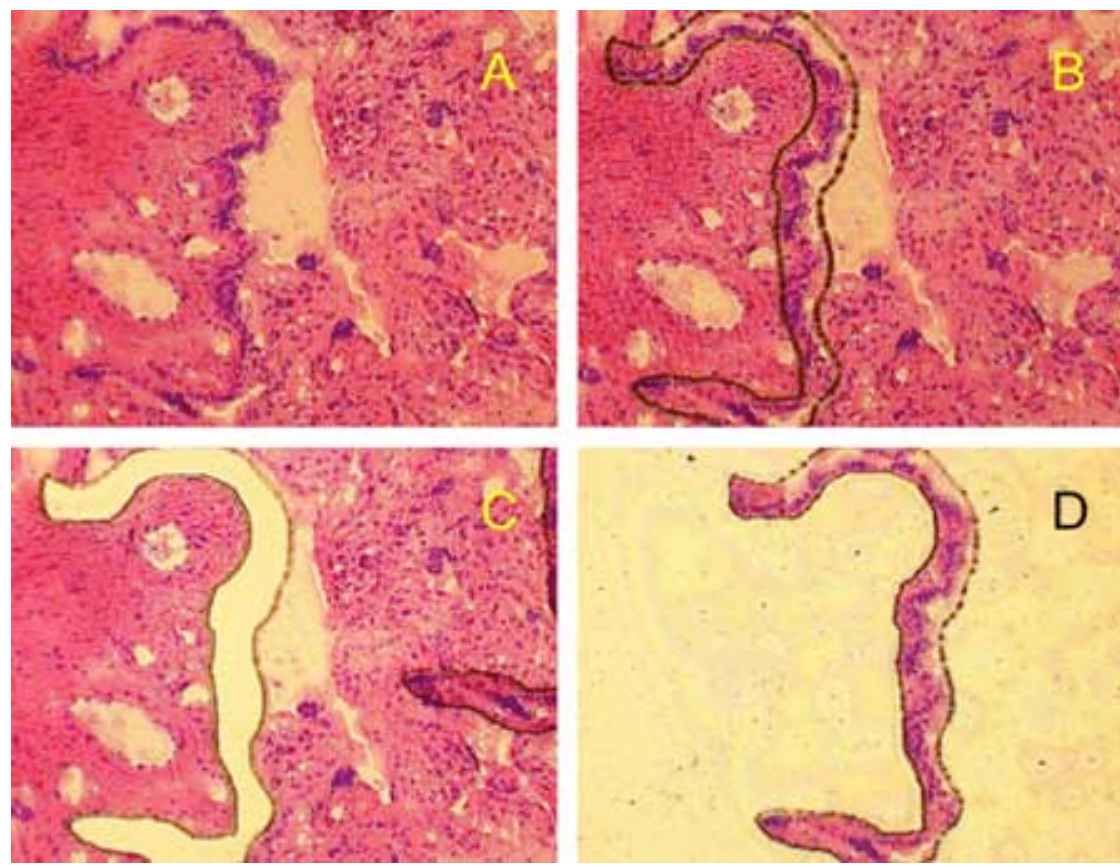

Figure 1

Representative image showing laser capture microdissection of human placental trophoblast. (A) Native placental tissue stained with haematoxylin and eosin. (B) Laser cut around trophoblast layer. Residual tissue (C) and trophoblast (D) capture. http://joe.endocrinology-journals.org DOI: $10.1530 / J O E-15-0424$
(C) 2016 Society for Endocrinology Printed in Great Britain 
NuGEN Direct Lysis Module before hybridisation on Affymetrix HuGene ST 1.0 array.

\section{Differential gene expression profiles of Norm, Ob, and GDM subjects}

Among the 28,869 transcript-level probes comprising the main design of the microarray, 329 probes exhibited ranked differential gene expression of at least two-fold change (up/down) between the subject groups. Of these, 130 transcripts were annotated with Entrez Gene ID (corresponding to 106 genes) and these were used for downstream functional enrichment analysis. We assessed the gene expression profile differences between Norm, Ob, and GDM subjects. We found 46 DEGs from Norm versus GDM comparison (Contrast 1); 23 genes from Norm versus $\mathrm{Ob}$ (Contrast 2); 70 genes from GDM vs $\mathrm{Ob}$ comparison (Contrast 3) (Fig. 2A). Of the DEGs from Contrast 1, 19 DEGs were unique to this pairwise comparison; these genes characterise GDM subjects who are also obese. We found that nine DEGs were unique to Contrast 2 i.e., Ob individuals compared with normal individuals. In contrast, 45 DEGs were unique to Contrast 3 wherein GDM individuals were subject-matched to obese individuals. As such, genes of interest from Contrast 3 characterise a state of GDM taking into account obesity as a confounder (Fig. 2A). We also found that 8 DEGs were common between Contrast 1 and Contrast 2. In addition, 6 DEGs were common between Contrast 2 and Contrast 3, whereas 19 genes were common between Contrast 1 and 3 (Fig. 2A). The list of DGEs identified from each pairwise comparison (Contrast 1-3) is found in Supplementary Table 1 (see section on supplementary data given at the end of this article). Probe level information including annotated GeneID and fold change in gene expression between subject groups are listed in Supplementary Table 2 .

Array intensities (after preprocessing and data normalization) of all probes for genes with at least twofold change (i.e., 391 probes) were visualized using a heatmap (Fig. 2B). As illustrated in the dendrogram on the horizontal axis of the heatmap, hierarchical sample clustering generated two main spot clusters. The
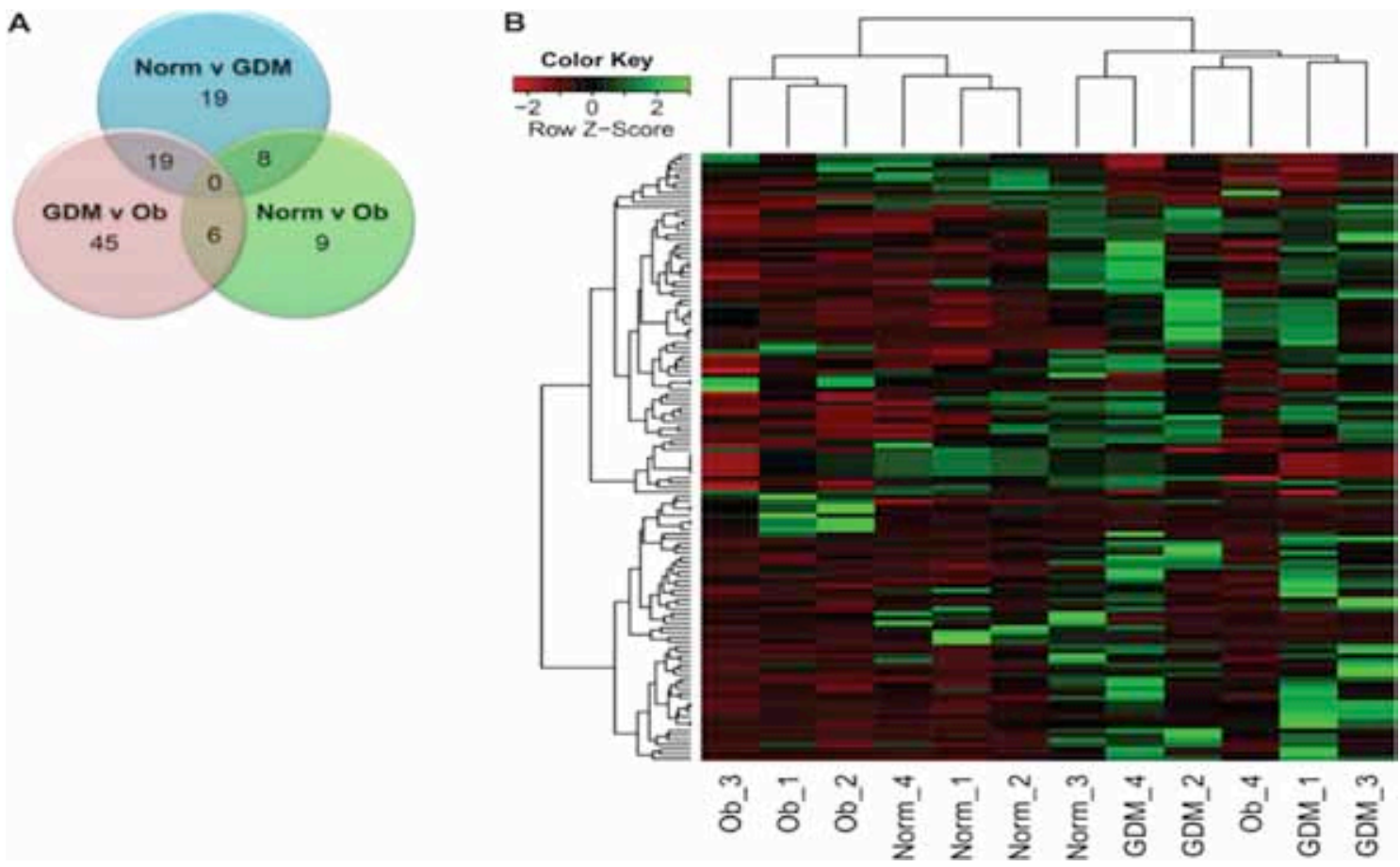

\section{Figure 2}

Gene expression profile characterising Norm, Ob, and GDM subjects. (A) The number of differentially expressed genes that are unique or common between Norm, Ob, and GDM pairwise comparisons. Contrast 1: Norm vs GDM; Contrast 2: Norm vs Ob; Contrast 3: GDM vs Ob. Criterion: $\geq 2$ ranked fold change (up/down) between any pairwise comparison. (B) Hierarchical clustering of subjects showing GDM as distinctly different from Norm and $\mathrm{Ob}$ individuals. Array intensities for probes with $\geq 2$-fold change (up/down) between any pairwise comparisons were centred and scaled (row z-score), and then used to generate the heatmap. Agglomeration method: complete linkage. Distance measure: Euclidean. Red-Black-Green color key denotes low to high gene expression. "_Integer" on the horizontal axis denotes individual subjects in each group.

http://joe.endocrinology-journals.org DOI: $10.1530 / \mathrm{JOE}-15-0424$
(C) 2016 Society for Endocrinology Printed in Great Britain
Published by Bioscientifica Ltd. 


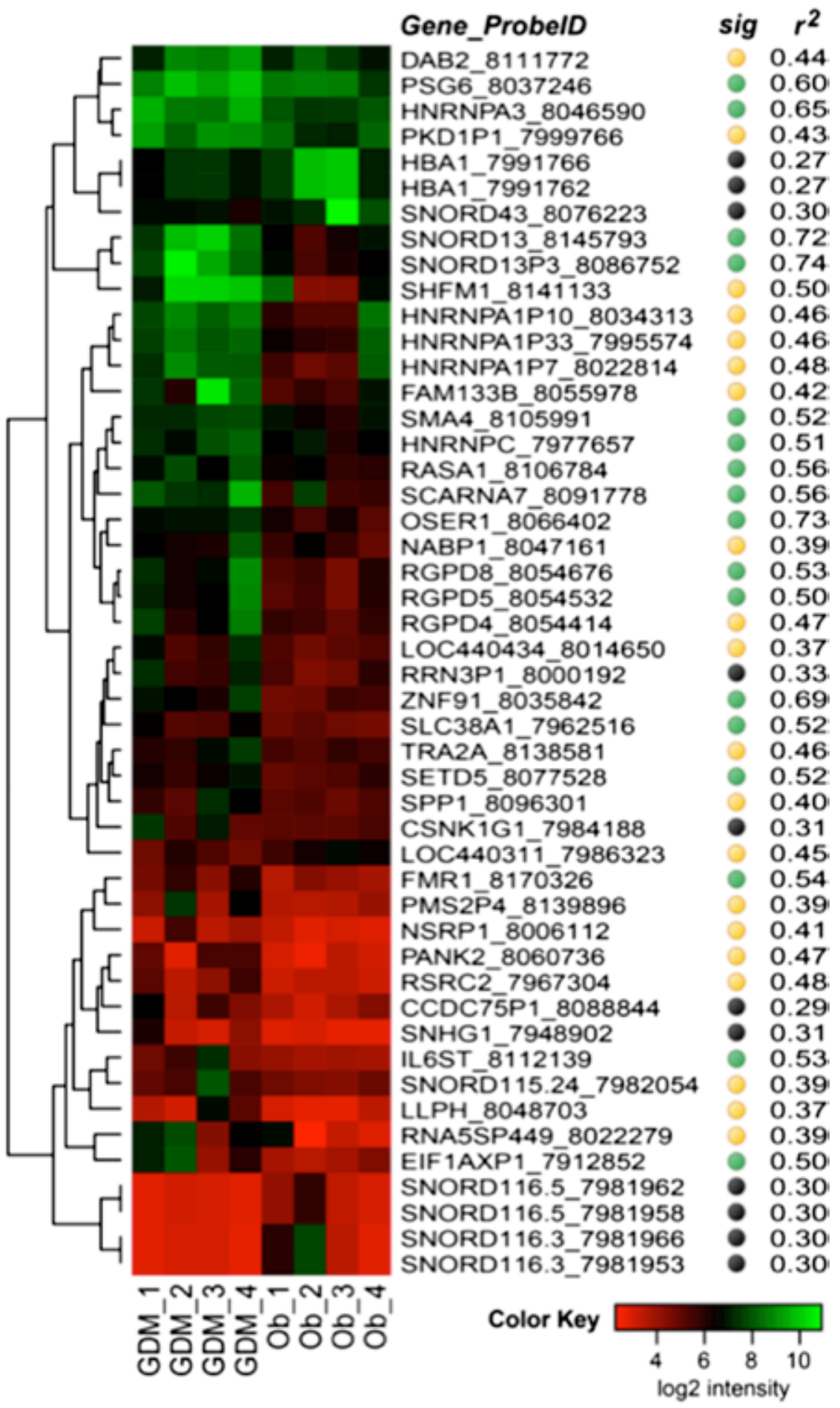

Figure 3

Genes differentially expressed in GDM are associated with maternal fasting plasma glucose. Left: Differentially expressed genes unique to GDM vs Ob comparison (Contrast 3) were clustered by expression level (log2 intensity). Agglomeration method used was complete linkage. Distance measure used was Euclidean. Genes are illustrated at the transcript level for individual subjects. Colour key: red-black-green represents low to high expression. 'Gene ProbelD' denotes Gene Symbol and corresponding Transcript Cluster Identifier from the microarray. Right: Results of the linear regression analysis to determine association between gene expression and fasting plasma glucose level post OGTT for Ob and GDM subjects using 'deltaGT'. 'deltaGT' was calculated as [Glucose $]_{2 \mathrm{~h}}-[\text { Glucose }]_{\text {Baseline }}$ measured by OGTT. 'sig' denotes level of significance from the regression analysis; Green: $P<0.05$, Yellow: $0.05<P<0.1$; Black: $P>0.1$. $r^{2}$ : coefficient of determination.
GDM subjects comprise one spot cluster, whereas the Norm and $\mathrm{Ob}$ subjects comprise the second larger spot cluster. Overall, the heatmap illustrates GDM subjects as a distinct group of individuals relative to Norm and Ob subjects based on their gene expression profiles. In support of this result, functional Gene Ontology term enrichment analysis showed that the significantly enriched GO terms for Contrast 1 and Contrast 2 were virtually identical and related to a wide range of processes including protein metabolic process, RNA processing, gene expression, and translational processes with the exception of GO:0022904 (respiratory electron

Published by Bioscientifica Ltd. 
transport chain) which was associated with Contrast 1 but not Contrast 2 . However, the significantly enriched GO terms for Contrast 3 are distinct from those of both Contrasts 1 and 2: enriched GO terms annotated to RNA splicing processes, intracellular transport of proteins to Golgi (Supplementary Table 3). Similarly, several KEGG pathways including oxidative phosphorylation (Kegg:00190) and ribosome (Kegg:03010) pathways were predicted as being significantly enriched in both Contrasts 1 and 2. However, only the spliceosome pathway (Kegg:03040) was significantly over-represented in Contrast 3 (Supplementary Table 4). Three miRNAs (hsa-miR-876-5p, hsa-miR-151-3p, hsa-miR-641) were predicted as being significantly over-represented in Contrast 3 (GDM), whilse no miRNA enrichment was found for Contrasts 1 and 2 (Supplementary Table 5), suggesting that these miRNAs may be the useful indicators of GDM taking into account obesity as the confounder.
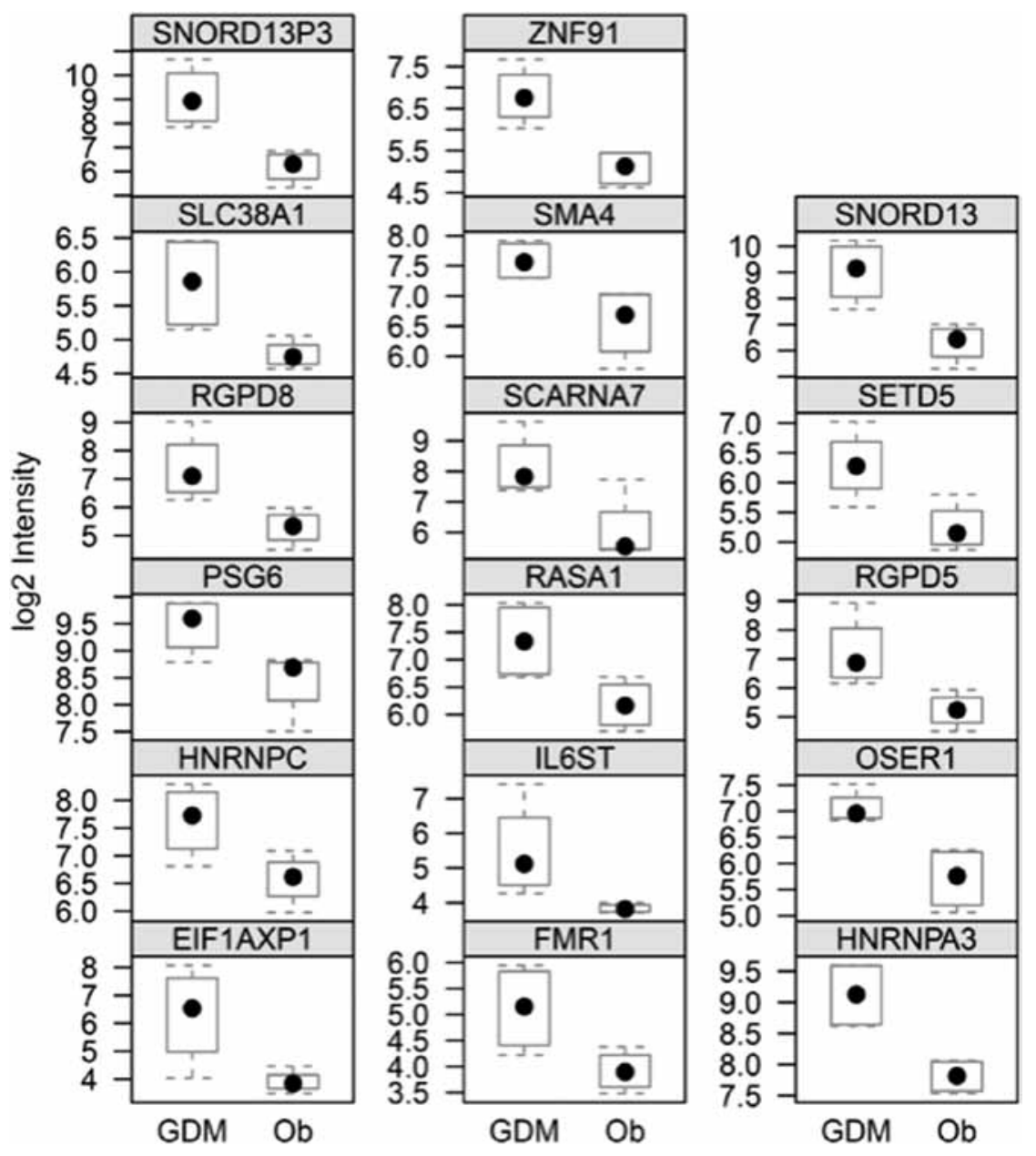

Figure 4

Increased expression of GDM-associated genes taking into account obesity as the confounder and which are significantly associated with maternal fasting plasma glucose. Expression levels of genes unique to Contrast 3 (GDM vs Ob). Box denotes 25 th to 75 th percentile, tails denote the range (maximum and minimum), and dot inside box denotes median expression. Gene names: EIF1AXP1: eukaryotic translation initiation factor $1 \mathrm{~A}, \mathrm{X}$-linked pseudogene 1 : FMR1: fragile $\mathrm{X}$ mental retardation 1 ; HNRNPA3 and HNRNPC: heterogeneous nuclear ribonucleoprotein $A 3$ and $C$ respectively; IL6ST: interleukin 6 signal transducer; OSER1: Oxidative Stress Responsive Serine-rich 1; PSG6: Pregnancy Specific beta-1-Glycoprotein 6; RASA1: RAS p21 protein activator (GTPase activating protein) $1 ; R G P D 5$ and $R G P D 8$ : RANBP2-like and GRIP domain containing 5 and 8 respectively; SCARNA7: small Cajal body-specific RNA 7; SETD5: SET domain containing 5; SLC38A1: solute carrier family 38, member 1; SMA4: glucuronidase, beta pseudogene; SNORD13: small nucleolar RNA, C/D box 13; SNORD13P3: small nucleolar RNA C/D box 13 pseudogene 3; ZNF91: zinc finger protein 91 . 
We also assessed the presence of differentially expressed splice variants between Norm, Ob, and GDM groups. We applied the two-fold change criterion to assess 'flmrna' probes (which are assigned to mRNA transcripts based on the Affymetrix annotation metafile). We found several differentially expressed transcript variants in Contrast 1 (Norm vs GDM), Contrast 2 (Norm vs Ob), and Contrast 3 (GDM vs Ob) (Table 2). Interestingly, transcript variants of ubiquitinconjugating enzymes (UBE2D3) and (UBE2V1) were heavily represented in Contrasts 1 and 3 . The levels of UBE2D3 transcript variants were increased in GDM group by 2.5 - to 3.0 -fold relative to Norm, and 2.8 to 3.3 -fold relative to Ob groups. Similarly, UBE2V1 transcript variant 4 level was increased in the GDM group by three-fold compared with Norm, and by fourfold compared with Ob group (Table 2). Distribution of the $\log 2$ intensities of these eight mRNA variants across the Norm, Ob, and GDM groups is shown in Fig. 5A. Microarray data were further confirmed by separate qRT-PCR (Supplementary Table 6). Furthermore, our regression analysis showed that increased maternal fasting plasma glucose (post OGTT) is significantly associated with increased levels of these eight UBE variants (Fig. $5 \mathrm{~B} ; P<0.05$ ). These results suggest that increased UBE2D3 and UBE2V1 expression is associated with maternal glucose intolerance, which characterizes a state of GDM.

Table 2 Differentially expressed mRNA transcripts in GDM compared with Norm or Ob subjects.

\begin{tabular}{|c|c|c|c|}
\hline Group & FC & mRNA_AccNo & mRNA_Name \\
\hline \multirow[t]{10}{*}{1 (Norm-v-GDM) } & -2.68 & NM_003340.4 & $\begin{array}{l}\text { Homo sapiens ubiquitin-conjugating enzyme E2D } 3 \text { (UBC4/5 homolog, yeast) } \\
\text { (UBE2D3), transcript variant 1, mRNA }\end{array}$ \\
\hline & -2.78 & NM_181887.1 & $\begin{array}{l}\text { Homo sapiens ubiquitin-conjugating enzyme E2D } 3 \text { (UBC4/5 homolog, yeast) } \\
\text { (UBE2D3), transcript variant 3, mRNA }\end{array}$ \\
\hline & -2.84 & NM_181888.1 & $\begin{array}{l}\text { Homo sapiens ubiquitin-conjugating enzyme E2D } 3 \text { (UBC4/5 homolog, yeast) } \\
\text { (UBE2D3), transcript variant 4, mRNA }\end{array}$ \\
\hline & -2.93 & NM_181889.1 & $\begin{array}{l}\text { Homo sapiens ubiquitin-conjugating enzyme E2D } 3 \text { (UBC4/5 homolog, yeast) } \\
\text { (UBE2D3), transcript variant 5, mRNA }\end{array}$ \\
\hline & -2.63 & NM_181890.1 & $\begin{array}{l}\text { Homo sapiens ubiquitin-conjugating enzyme E2D } 3 \text { (UBC4/5 homolog, yeast) } \\
\text { (UBE2D3), transcript variant 6, mRNA }\end{array}$ \\
\hline & -3.01 & NM_181891.1 & $\begin{array}{l}\text { Homo sapiens ubiquitin-conjugating enzyme E2D } 3 \text { (UBC4/5 homolog, yeast) } \\
\text { (UBE2D3), transcript variant 7, mRNA }\end{array}$ \\
\hline & -2.47 & NM_181893.1 & $\begin{array}{l}\text { Homo sapiens ubiquitin-conjugating enzyme E2D } 3 \text { (UBC4/5 homolog, yeast) } \\
\text { (UBE2D3), transcript variant 9, mRNA }\end{array}$ \\
\hline & -2.21 & NM_001187.1 & Homo sapiens B melanoma antigen (BAGE), mRNA \\
\hline & 2.07 & NM_001029945.1 & $\begin{array}{l}\text { Homo sapiens chromosome } 1 \text { open reading frame } 178 \text { (C1orf178), transcript } \\
\text { variant } c \text {, mRNA }\end{array}$ \\
\hline & -3.26 & NM_001032288.1 & $\begin{array}{l}\text { Homo sapiens ubiquitin-conjugating enzyme E2 variant } 1 \text { (UBE2V1), transcript } \\
\text { variant } 4 \text {, mRNA }\end{array}$ \\
\hline \multirow[t]{2}{*}{2 (Norm-v-Ob) } & 2.10 & NM_145326.1 & Homo sapiens similar to hypothetical protein FLJ13659 (LOC115648), mRNA \\
\hline & 2.09 & NM_001014450.1 & Homo sapiens small proline-rich protein $2 \mathrm{~F}(S P R R 2 F)$, mRNA \\
\hline \multirow[t]{9}{*}{3 (GDM-v-Ob) } & 3.01 & NM_145326.1 & Homo sapiens similar to hypothetical protein FLJ13659 (LOC115648), mRNA \\
\hline & 3.08 & NM_003340.4 & $\begin{array}{l}\text { Homo sapiens ubiquitin-conjugating enzyme E2D } 3 \text { (UBC4/5 homolog, yeast) } \\
\text { (UBE2D3), transcript variant 1, mRNA }\end{array}$ \\
\hline & 2.94 & NM_181887.1 & $\begin{array}{l}\text { Homo sapiens ubiquitin-conjugating enzyme E2D } 3 \text { (UBC4/5 homolog, yeast) } \\
\text { (UBE2D3), transcript variant } 3 \text {, mRNA }\end{array}$ \\
\hline & 3.15 & NM_181888.1 & $\begin{array}{l}\text { Homo sapiens ubiquitin-conjugating enzyme E2D } 3 \text { (UBC4/5 homolog, yeast) } \\
\text { (UBE2D3), transcript variant 4, mRNA }\end{array}$ \\
\hline & 3.23 & NM_181889.1 & $\begin{array}{l}\text { Homo sapiens ubiquitin-conjugating enzyme E2D } 3 \text { (UBC4/5 homolog, yeast) } \\
\text { (UBE2D3), transcript variant 5, mRNA }\end{array}$ \\
\hline & 2.84 & NM_181890.1 & $\begin{array}{l}\text { Homo sapiens ubiquitin-conjugating enzyme E2D } 3 \text { (UBC4/5 homolog, yeast) } \\
\text { (UBE2D3), transcript variant 6, mRNA }\end{array}$ \\
\hline & 3.30 & NM_181891.1 & $\begin{array}{l}\text { Homo sapiens ubiquitin-conjugating enzyme E2D } 3 \text { (UBC4/5 homolog, yeast) } \\
\text { (UBE2D3), transcript variant 7, mRNA }\end{array}$ \\
\hline & 2.77 & NM_181893.1 & $\begin{array}{l}\text { Homo sapiens ubiquitin-conjugating enzyme E2D } 3 \text { (UBC4/5 homolog, yeast) } \\
\text { (UBE2D3), transcript variant 9, mRNA }\end{array}$ \\
\hline & 4.16 & NM_001032288.1 & $\begin{array}{l}\text { Homo sapiens ubiquitin-conjugating enzyme E2 variant } 1 \text { (UBE2V1), transcript } \\
\text { variant } 4 \text {, mRNA }\end{array}$ \\
\hline
\end{tabular}

FC denotes fold change in expression. Positive FC indicates increased expression in Norm for Contrasts 1 and 2, and increased expression in GDM for Contrast 3. mRNA_AccNo denotes GenBank Accession Number.

http://joe.endocrinology-journals.org DOI: 10.1530/JOE-15-0424
(C) 2016 Society for Endocrinology Printed in Great Britain
Published by Bioscientifica Ltd. 
A

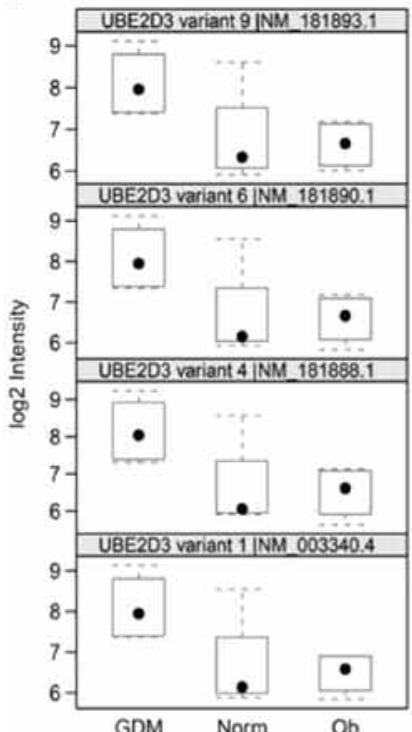

UBE2V1 variant 4 INM 001032288.1

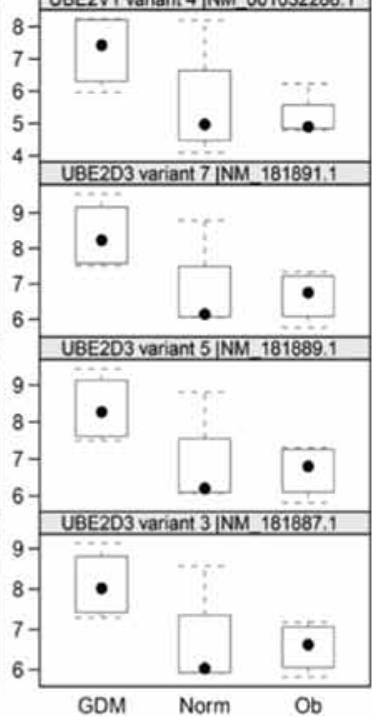

B

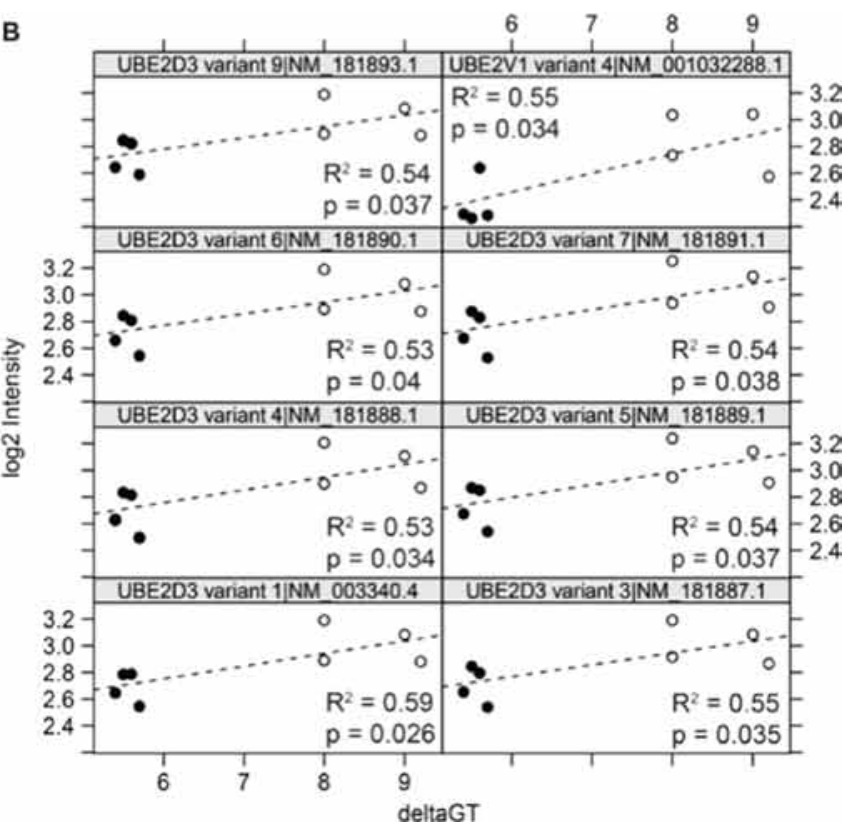

Figure $\mathbf{5}$

Increased expression of mRNA variants of ubiquitin-conjugating enzymes which discriminates GDM phenotype from Ob and Norm is positively correlated with maternal OGTT. (A) Box denotes 25th to 75th percentile; tail denotes the range (maximum and minimum); dot inside box denotes median expression. Norm: Normal; Ob: Obese; GDM: Gestational diabetic. (B) Scatter plot of UBE variants (log2 intensity) versus fasting plasma

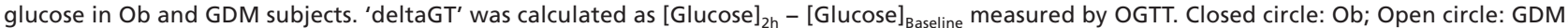
Panel title: 'UBE variant name|Accession number'.

\section{Discussion}

The impact of GDM on the progeny is made difficult to assess because of the confounding influence of maternal obesity in the manifestation of foetal phenotypes. The strength of the current study is the inclusion of obese subjects for comparison with GDM subjects with the aim of more accurately defining the molecular basis for disturbed placental function in GDM pregnancies. Indeed, our findings suggest that genes involved in RNA splicing processes and the intracellular transport of proteins to the Golgi apparatus may be differentially expressed in GDM subjects compared with normal or obese subjects, implying that GDM may be fundamentally distinct from states of normality or obesity at the molecular level.

Additional strengths include careful selection of patient to exclude other pathologies that might have impacted data analysis, optimization of RNA integrity using a variety of commonly used stains, and finally using patients who had undergone elective Caesarean section, which excluded potential stresses placed on the placenta by labour (Reddy et al. 2008).

A further strength is our approach of using LCM to investigate the changes in placental trophoblast gene expression. As the placenta is a heterogeneous organ (Huppertz 2008), our approach of using LCM facilitates molecular analysis of trophoblast without contamination from other cell types. In this study, we present an optimized protocol for RNA extraction using LCM, which demonstrates that RNA integrity (RIN) is sustained when staining with alcoholic cresyl violet and alcoholic eosin-Y (Clement-Ziza et al. 2008). Moreover, we demonstrate that LCM can be performed with this stain for up to 30 min per slide with no significant degradation of RNA. We note that other publications using laser capture of placenta (Kim et al. 2013b) have used haematoxylin and eosin (which we have shown does not stabilize RNA) without demonstrating any formal assessment of RIN before hybridisation.

GDM is linked to placental function, and a number of placenta-derived molecules in particular from the trophoblast have been implicated in causing insulin resistance in GDM (Desoye and Hauguel-de Mouzon 2007). The expression of pregnancy-specific 1 glycoprotein (PSG), a common placental factor produced by trophoblast to allow for a viable pregnancy, has been reported to increase in preeclampsia (also known to be associated with increased insulin resistance (Catalano

Published by Bioscientifica Ltd. 
et al. 2012) and GDM (Okazaki et al. 2007, Tamsen et al. 1983)). Increased PSG levels are associated with elevated carbohydrate levels in humans with GDM (Neufeld et al. 1984). Consistent with this, we found increased PSG6 expression in GDM group compared with $\mathrm{Ob}$ group. In addition, increased PSG6 expression in our GDM subjects significantly associated with increased maternal fasting plasma glucose as a result of impaired glucose tolerance. Moreover, PSG6 and PSG11 genotypes have recently been found to be located with a genomic region enriched in segmental duplication in preeclampsia patients (Zhao et al. 2012) (a disease, perhaps suggesting a linkage between PSG6 and GDM and preeclampsia). Further studies into how glucose modulates placental PSG6 function may improve our understanding of the pathogenesis of GDM.

The trophoblast cell layer, in direct contact with maternal blood, is the transporting epithelium of the human placenta integrating foetal and maternal signals to modulate placental growth in response to alterations in maternal nutrient supply (Jansson \& Powell 2013). The placental system A sodium-dependent transporter system, comprising of the members SNAT1 (SLC38A1), SNAT2 (SLC38A2), and SNAT4 (SLC38A4), is important for supplying neutral amino acids necessary for foetal growth (Jansson \& Powell 2013). In addition, SLC38A1 is a key contributor to System A activity in term placenta (Desforges et al. 2010). The molecular mechanisms by which diabetes in pregnancy affects placental nutrient transport is still unclear; however Jansson and coworkers reported that System A-mediated amino acid transport activity is significantly increased in the trophoblast microvillous plasma membrane (MVM) in GDM, and the authors suggested the increase to be related to increased amino acid catabolism in the placenta (Jansson et al. 2002). In another study, SLC38A1 mRNA levels were found to be unchanged in placentae from preeclampsia, intrauterine growth-restricted, and small-for-gestationalage pregnancies i.e., models of intrauterine undernutrition. The authors concluded that regulation might occur at the transporter expression level or activity (Malina et al. 2005). Indeed, other studies found decreased System A transport activity in the MVM of intrauterine growth compared with the MVM of normal foetal growth (Glazier et al. 1997, Jansson et al. 2002). Our findings of increased SLC38A1 expression in trophoblast (Fig. 3) are in agreement with Jansson and coworkers study who reported increased transport activity with this transporter in the MVM from GDM pregnancies.

The novel finding of increased expression of ubiquitin-conjugating enzyme UBE2D3 and UBE2V1 transcript variants in GDM is intriguing. The ubiquitinproteasome system is multi-functional (Lecker et al. 2006); it is involved in rapid removal of unwanted or misfolded proteins (Lecker et al. 2006) and tightly regulates gene transcription (Dhananjayan et al. 2005, Lecker et al. 2006) and immunological processes (Lecker et al. 2006). Ubiquitin-conjugating enzymes (UBE2 variants facilitate the binding of ubiquitin molecules to ubiquitin ligases (UBE3 variants) to target a protein for proteasomedependent degradation (David et al. 2010). A recent study has reported the potential association of UBE2E2 polymorphisms with fasting plasma glucose and GDM (Kim et al. 2013a). Furthermore, UBE2 variants D1 and V2 have been recently found to be significantly differentially methylated in 8 to 12 year-old children exposed to maternal GDM in utero (West et al. 2013). Our finding and the emerging literature collectively suggest a link between UBE function and fasting plasma glucose in GDM.

The pattern of the DEGs we observed suggests that the ubiquitin-conjugating system is functionally more active in GDM compared with $\mathrm{Ob}$, implying greater turnover rates of translation products. Interestingly, a recent proteomics study has reported accumulation of misfolded proteins in the urine of preeclampsia subjects, thus implying compromised proteasome function (Buhimschi et al. 2014). Given that ubiquitination and the proteasomal system are tightly coupled, it is conceivable that perturbed regulation of protein turnover may underlie the pathogenesis of common pregnancy disorders such as GDM and preeclampsia. That these phenotypes may share the elements of common aetiology are perhaps not entirely unexpected. A considerable literature describes a propensity for maternal GDM to be associated with postnatal occurrence of offspring insulin resistance (reviewed in (Borgono et al. 2012)). Similarly, mid-trimester maternal insulin resistance has been identified as a possible potentiating factor in the incidence of preeclampsia (Hauth et al. 2011). Furthermore, the incidence of preeclampsia has also been identified as an harbinger of infant type 2 diabetes (Libby et al. 2007). Collectively, these observations suggest that maternal insulin resistance in pregnancy increases the likelihood of preeclampsia complications at parturition, and that both clinical features are strongly associated with the subsequent emergence of metabolic syndrome in the infant. Our findings warrant expansion into larger studies using populations representing wide multi-cohort comparisons, and encourage the development of appropriate in vitro models to functionally validate the relevance of the emerging candidate genes in a GDM context.

Published by Bioscientifica Ltd 
Supplementary data

This is linked to the online version of the paper at http://dx.doi.org/10.1530/ JOE-15-0424.

\section{Declaration of Interest}

The authors declare that there is no conflict of interest that could be perceived as prejudicing the impartiality of the research reported.

\section{Funding}

This study was supported by the National Institutes of Health (NIH) DK81412 (B.C.C).

\section{Author contribution statement}

$\mathrm{MV}$ and A M S originated the concept and designed the study. M B F and C C B optimized the laser capture methodology, and carried out the experiments. M B F, C C B and S N performed the microarray data analysis and participated in the drafting of the manuscript. All authors participated in the critical interpretation of the results, reviewed, and approved the final version of the manuscript.

\section{References}

Bari MF, Brown H, Nicholson AG, Kerr KM, Gosney JR, Wallace WA, Soomro I, Muller S, Peat D, Moore JD et al. 2013 BAI3, CDX2 and VIL1: a panel of three antibodies to distinguish small cell from large cell neuroendocrine lung carcinomas. Histopathology 64 547-556. (doi:10.1111/his.12278)

Bellamy L, Casas JP, Hingorani AD \& Williams D 2009 Type 2 diabetes mellitus after gestational diabetes: a systematic review and metaanalysis. Lancet 373 1773-1779. (doi:10.1016/S0140-6736(09)60731-5)

Boney CM, Verma A, Tucker R \& Vohr BR 2005 Metabolic syndrome in childhood: association with birth weight, maternal obesity, and gestational diabetes mellitus. Pediatrics 115 e290-e296.

Borgono CA, Hamilton JK, Ye C, Hanley AJ, Connelly PW, Sermer M, Zinman B \& Retnakaran R 2012 Determinants of insulin resistance in infants at age 1 year: impact of gestational diabetes mellitus. Diabetes Care 35 1795-1797. (doi:10.2337/dc12-0173)

Bottalico JN 2007 Recurrent gestational diabetes: risk factors, diagnosis, management, and implications. Seminars in Perinatology 31 176-184.

Bryson CL, Ioannou GN, Rulyak SJ \& Critchlow C 2003 Association between gestational diabetes and pregnancy-induced hypertension. American Journal of Epidemiology 158 1148-1153.

Buhimschi IA, Nayeri UA, Zhao G, Shook LL, Pensalfini A, Funai EF, Bernstein IM, Glabe CG \& Buhimschi CS 2014 Protein misfolding, congophilia, oligomerization, and defective amyloid processing in preeclampsia. Science Translational Medicine $6245 \mathrm{ra} 292$. (doi:10.1126/scitranslmed.3008808)

Burton GJ, Sebire NJ, Myatt L, Tannetta D, Wang YL, Sadovsky Y, Staff AC \& Redman CW 2014 Optimising sample collection for placental research. Placenta 35 9-22. (doi:10.1016/j.placenta.2013.11.005)

Carvalho B, Bengtsson H, Speed TP \& Irizarry RA 2007 Exploration, normalization, and genotype calls of high-density oligonucleotide SNP array data. Biostatistics 8 485-499.

Catalano PM, McIntyre HD, Cruickshank JK, McCance DR, Dyer AR, Metzger BE, Lowe LP, Trimble ER, Coustan DR, Hadden DR et al.
2012 The hyperglycemia and adverse pregnancy outcome study: associations of GDM and obesity with pregnancy outcomes. Diabetes Care 35 780-786. (doi:10.2337/dc11-1790)

Clement-Ziza M, Munnich A, Lyonnet S, Jaubert F \& Besmond C 2008 Stabilization of RNA during laser capture microdissection by performing experiments under argon atmosphere or using ethanol as a solvent in staining solutions. RNA 14 2698-2704.

Dabelea D, Snell-Bergeon JK, Hartsfield CL, Bischoff KJ, Hamman RF \& McDuffie RS 2005 Increasing prevalence of gestational diabetes mellitus (GDM) over time and by birth cohort: Kaiser Permanente of Colorado GDM Screening Program. Diabetes Care 28 579-584.

David Y, Ziv T, Admon A \& Navon A 2010 The E2 ubiquitinconjugating enzymes direct polyubiquitination to preferred lysines. Journal of Biological Chemistry 285 8595-8604. (doi:10.1074/jbc.M109.089003)

Desforges M, Greenwood SL, Glazier JD, Westwood M \& Sibley CP 2010 The contribution of SNAT1 to system A amino acid transporter activity in human placental trophoblast. Biochemical and Biophysical Research Communications 398 130-134. (doi: 10.1016/ j.bbrc.2010.06.051)

Desoye G \& Hauguel-de Mouzon S 2007 The human placenta in gestational diabetes mellitus. The insulin and cytokine network. Diabetes Care 30 (Supplement 2) S120-S126. (doi: 10.2337/ dc07-s203)

Dhananjayan SC, Ismail A \& Nawaz Z 2005 Ubiquitin and control of transcription. Essays in Biochemistry 41 69-80.

Glazier JD, Cetin I, Perugino G, Ronzoni S, Grey AM, Mahendran D, Marconi AM, Pardi G \& Sibley CP 1997 Association between the activity of the system A amino acid transporter in the microvillous plasma membrane of the human placenta and severity of fetal compromise in intrauterine growth restriction. Pediatric Research 42 514-519.

Hauth JC, Clifton RG, Roberts JM, Myatt L, Spong CY, Leveno KJ, Varner MW, Wapner RJ, Thorp JM, Jr., Mercer BM et al. 2011 Maternal insulin resistance and preeclampsia. American Journal of Obstetrics \& Gynecology 204327 e321-e326.

Huppertz B 2008 The anatomy of the normal placenta. Journal of Clinical Pathology 61 1296-1302.

Irizarry RA, Hobbs B, Collin F, Beazer-Barclay YD, Antonellis KJ, Scherf U \& Speed TP 2003 Exploration, normalization, and summaries of high density oligonucleotide array probe level data. Biostatistics 4 249-264.

Jansson T \& Powell TL 2013 Role of placental nutrient sensing in developmental programming. Clinical Obstetrics and Gynecology 56 591-601. (doi:10.1097/GRF.0b013e3182993a2e)

Jansson T, Ekstrand Y, Bjorn C, Wennergren M \& Powell TL 2002 Alterations in the activity of placental amino acid transporters in pregnancies complicated by diabetes. Diabetes 51 2214-2219.

Kim JY, Cheong HS, Park BL, Baik SH, Park S, Kim S, Shin HD \& Kim SH 2013a Putative association between UBE2E2 polymorphisms and the risk of gestational diabetes mellitus. Gynecological Endocrinology 29 904-908. (doi:10.3109/09513590.2013.813465)

Kim SH, Shim SH, Sung SR, Lee KA, Shim JY, Cha DH \& Lee KJ 2013b Gene expression analysis of the microdissected trophoblast layer of human placenta after the spontaneous onset of labor. PLoS One $\mathbf{8}$ e77648. (doi:10.1371/journal.pone.0077648)

Lecker SH, Goldberg AL \& Mitch WE 2006 Protein degradation by the ubiquitin-proteasome pathway in normal and disease states. Journal of the American Society of Nephrology 17 1807-1819.

Libby G, Murphy DJ, McEwan NF, Greene SA, Forsyth JS, Chien PW, Morris AD \& Collaboration DM 2007 Pre-eclampsia and the later development of type 2 diabetes in mothers and their children: an intergenerational study from the Walker cohort. Diabetologia 50 523-530. 
Malina A, Daftary A, Crombleholme W, Markovic N \& Roberts JM 2005 Placental system A transporter mRNA is not different in preeclampsia, normal pregnancy, or pregnancies with small-forgestational-age infants. Hypertens Pregnancy 24 65-74.

Neufeld N, Braunstein G, Corbo LM, Gratacos J \& Mestman J 1984 Insulin receptors and placental proteins in normal and gestational-diabetic pregnancies. Biological Research in Pregnancy and Perinatology 5 84-89.

Okazaki S, Sekizawa A, Purwosunu Y, Farina A, Wibowo N \& Okai T 2007 Placenta-derived, cellular messenger RNA expression in the maternal blood of preeclamptic women. Obstetrics \& Gynecology 110 1130-1136.

Reddy A, Zhong XY, Rusterholz C, Hahn S, Holzgreve W, Redman CW \& Sargent IL 2008 The effect of labour and placental separation on the shedding of syncytiotrophoblast microparticles, cell-free DNA and mRNA in normal pregnancy and pre-eclampsia. Placenta 29 942-949. (doi:10.1016/ j.placenta.2008.08.018)

Smyth GK 2004 Linear models and empirical bayes methods for assessing differential expression in microarray experiments. Statistical Applications in Genetics and Molecular Biology 3 Article3.
Tamsen L, Axelsson O \& Johansson SG 1983 Serum levels of pregnancyspecific beta 1-glycoprotein (SP1) in women with pregnancies at risk. Gynecologic and Obstetric Investigation 16 253-260.

Virjee S, Robinson S \& Johnston DG 2001 Screening for diabetes in pregnancy. Journal of the Royal Society of Medicine 94 502-509.

Vohr BR \& Boney CM 2008 Gestational diabetes: the forerunner for the development of maternal and childhood obesity and metabolic syndrome? Journal of Maternal-Fetal and Neonatal Medicine 21 149-157. (doi:10.1080/14767050801929430)

Wang Q, Huang R, Yu B, Cao F, Wang H, Zhang M, Wang X, Zhang B, Zhou H \& Zhu Z 2013 Higher fetal insulin resistance in Chinese pregnant women with gestational diabetes mellitus and correlation with maternal insulin resistance. PLoS One 8 e59845. (doi:10.1371/journal.pone.0059845)

West NA, Kechris K \& Dabelea D 2013 Exposure to maternal diabetes in utero and DNA methylation patterns in the offspring. Immunometabolism 1 1-9.

Zhao L, Triche EW, Walsh KM, Bracken MB, Saftlas AF, Hoh J \& Dewan AT 2012 Genome-wide association study identifies a maternal copynumber deletion in PSG11 enriched among preeclampsia patients. BMC Pregnancy Childbirth 1261.

Received in final form 4 February 2016

Accepted 11 February 2016

Accepted Preprint published online 11 February 2016
() 2016 Society for Endocrinology Printed in Great Britain
Published by Bioscientifica Ltd. 